\title{
DOES PALM OIL VITAMIN E REDUCE THE RISK OF PREGNANCY INDUCED HYPERTENSION?
}

\author{
Zaleha Abdullah Mahdy', Harlina Halizah Siraj', Huzwah Khaza'ai', Mohd Sokhini Abdul Mutalib ${ }^{3}$, \\ Muhammad Hatta Azwar', Marianah Abdul Wahab', Ahmad Zailani Hatta Md Dali', Rohana Jaafar', \\ Nor Azlin Mohd Ismail ${ }^{1}$, Muhammad Abdul Jamil ${ }^{1}$, Nafisah Adeeb
}

Faculty of Medicine, Universiti Kebangsaan Malaysia, Jalan Yaacob Latiff, 56000 Cheras, Kuala Lumpur, Malaysia: Department of Obstetrics and Gynaecology ${ }^{1}$, Department of Paediatrics ${ }^{4}$; Universiti Putra Malaysia, Faculty of Medicine and Health Sciences, Serdang, 43400 Selangor, Malaysia: Department of Biomedical Sciences ${ }^{2}$, Department of Nutrition and Dietetics ${ }^{3}$

Summary: In view of the high anti-oxidative potential of tocotrienol, the role of the tocotrienol-rich fraction (TRF) of palm oil in preventing pregnancy induced hypertension (PIH) was explored in a randomized double-blind placebo-controlled clinical trial in an urban teaching hospital. Healthy primigravidae were randomized to receive either oral TRF 100mg daily or placebo, from early second trimester until delivery. Out of 299 women, 151 were randomized into the TRF arm and 148 into the placebo arm. A total of $15(5.0 \%)$ developed PIH. Although there was no statistically significant difference in the incidence of PIH (4/151 or $2.6 \%$ in the TRF arm vs $11 / 148$ or $7.4 \%$ in the placebo arm, $p=0.058)$ between the two arms, there was a tendency towards a lower incidence of PIH in the TRF arm compared to the placebo arm. With TRF supplementation, the relative risk (RR) of PIH was 0.36 (95\% CI 0.12-1.09). In conclusion, although TRF from palm oil does not statistically significantly reduce the risk of development of PIH in the population studied, the $64 \%$ reduction in incidence of PIH is substantial. The findings warrant further clinical trials, particularly in high risk populations.

Keywords: Preeclampsia prevention; Randomized controlled trial; Palm oil; Tocotrienol

\section{Introduction}

Pregnancy induced hypertension (PIH), specifically preeclampsia, is reported to affect between $0.4 \%$ and $3.6 \%$ of pregnancies in developed regions of the world $(1,2)$, with a higher prevalence in less developed countries (3). It occurs most commonly in first pregnancies with a prevalence of about 5\%, and carries the highest maternal and fetal morbidity and mortality of all pregnancy complications, with more than $90 \%$ of the most serious outcomes occurring in developing countries (1).

The International Society for the Study of Hypertension in Pregnancy (ISSHP) defines preeclampsia as PIH (diastolic blood pressure $>90 \mathrm{mmHg}$ ) occurring after 20 weeks gestation with proteinuria (either $\geq 300 \mathrm{mg}$ protein per day or a urinary protein/creatinine ratio $\geq 30 \mathrm{mg} / \mathrm{mmol}$ ) (4). PIH is a broader term that encompasses both preeclampsia and gestational hypertension, the non-proteinuric counterpart of preeclampsia. The appearance of proteinuria, i.e. development of preeclampsia, heralds worsening of a disease that has a wide spectrum of manifestation and severity.

Although the cause of preeclampsia and PIH remains largely unknown, poor placentation is believed to play an important role. Several theories are proposed to link this phenomenon to the resulting maternal syndrome. A pivotal role of enhanced placental superoxide generation, leading to oxidative stress that damages maternal systemic vascular endothelial function, is increasingly recognized $(5,6)$.

Preeclampsia is postulated to originate primarily in failure of the second wave of trophoblastic invasion into the myometrial stroma, which normally occurs between the twelfth and sixteenth weeks of pregnancy. It is hypothesized that this localized defect in placentation causing placental ischemia is amplified into the systemic maternal syndrome via release of circulating factors which in turn leads to maternal endothelial cell dysfunction in preeclampsia (7).

An intriguing possibility was raised by Hubel et al. (8), who suggested lipid peroxides as the putative noxious agent. These substances are now known to be elevated in the blood of preeclamptic women (9) and have many of the properties predicted for the postulated agent. The proposed model is centered on the concept that a derangement in the oxidant-antioxidant balance intensifies lipid peroxidation leading to dysfunction of the defensive vasodilatory and anti-aggregatory activities of the vascular endothelium. This in turn contributes to increased peripheral resistance and pressor reactivity to vasoagonists.

At the time when this study was proposed, there was only one randomized controlled trial using antioxidants including vitamin $\mathrm{E}$ in the form of tocopherol in the treatment of established severe preeclampsia in South Africa, but it failed to demonstrate any beneficial effect (10). The authors, 
however, admitted that treatment may have been started too late in their study.

Vitamin E is a potent antioxidant, particularly in the form of tocotrienol (11). Palm oil is especially rich in tocotrienol, and the vitamin E preparation derived from palm oil is known as the tocotrienol rich fraction (TRF) of this natural product. This study was therefore designed to look at the effect of early supplementation with TRF on the subsequent incidence of $\mathrm{PIH}$ in a group of primigravidae with no other risk factors for the disease. The NICE guideline now recognizes primigravidity as a moderate risk factor for preeclampsia (12).

\section{Material and Methods}

\section{Study Design}

The study was a randomized placebo-controlled double blind clinical trial. The software Statview 4.0 was used to generate random numbers.

\section{Inclusion Criteria}

All normotensive non-proteinuric primigravidae with singleton pregnancies on follow-up at the antenatal clinic of the Universiti Kebangsaan Malaysia Medical Centre (UKMMC), between 12 and 16 weeks gestation, were eligible for recruitment into the study.

\section{Exclusion Criteria}

Women with pre-existing hypertension \pm proteinuria, or multiple pregnancy, or chronic liver disease or lipid malabsorption, or known allergy to vitamin E, or on anti-coagulant therapy, or refused entry into the trial, were excluded.

\section{Sample Size}

The incidence of PIH (preeclampsia plus gestational hypertension) was assumed to be twice the incidence of preeclampsia alone, i.e. $10 \%$. To demonstrate a statistically significant reduction in the incidence of PIH from $10 \%$ to $2 \%$, a sample size of 135 is required on each arm at $95 \%$ confidence interval with a power of $80 \%$.

To ensure similar distribution of maternal body weight on the two arms of the trial, randomization was further blocked into series A and B according to maternal body weight at booking. A body weight of $55 \mathrm{~kg}$ was the median body weight at booking at 12-16 weeks gestation according to a pre-study survey of 30 consecutive patients at our clinic. Subjects with body weights of less than $55 \mathrm{~kg}$ were therefore randomized according to numbers in series A, whereas those with body weights of $55 \mathrm{~kg}$ or more were randomized according to numbers in series $\mathrm{B}$.

\section{TRF and Placebo}

A daily supplement of 100mg TRF (Tri-E ${ }^{\circledR}$, Golden Hope Bioganics, Kuala Lumpur, Malaysia), in super olein capsules, was given to subjects in the trial arm from about 12 to 16 weeks pregnancy until delivery. The placebo arm was given super olein capsules without TRF over the same duration of time. The TRF and placebo capsules appear similar and not easily distinguishable from each other.

\section{Ethical Approval}

The study was performed according to the Declaration of Helsinki, and approval from the institutional research ethics committee was obtained prior to commencement of the study. Written consent was obtained from all patients prior to recruitment and randomization.

\section{Maternal Monitoring}

Subjects were seen at booking (between 12 to 16 weeks), and subsequently at 20,24,28,32,36, 38 and 40 weeks of pregnancy. At each visit, maternal blood pressure was measured and urinalysis was conducted to look for proteinuria. Any other nutritional supplements taken by the patient either

Tab. 1: Socio-demographic profile of subjects in the TRF arm compared to the placebo arm

\begin{tabular}{|l|l|c|c|c|}
\hline Socio-demographic Characteristic & & TRF Arm (n=151) & Placebo Arm $(\mathrm{n}=148)$ & $p$ \\
\hline Age (years) * & & $25.94 \pm 0.303$ & $25.77 \pm 0.295$ & 0.687 \\
\hline Body weight (kg)* & & $55.27 \pm 1.076$ & $55.58 \pm 0.867$ & 0.823 \\
\hline \multirow{2}{*}{ Ethnicity, n (\%) } & Malay & $112(74.2)$ & $111(75.0)$ & $26(17.6)$ \\
& Chinese & $26(17.2)$ & $7(4.7)$ \\
& Indian & $12(7.9)$ & $4(2.7)$ & \\
\hline \multirow{2}{*}{ Employment Status, n (\%) } & Others & $134(8.7)$ & $137(92.6)$ & $11(7.4)$ \\
\hline Household Income (RM / month)** & Employed & $17(11.3)$ & $1001-3000$ & \\
\hline Gestation at recruitment (weeks)* & & $1001-3000$ & $13.64 \pm 0.079$ & 0.458 \\
\hline
\end{tabular}

* Mean \pm SEM; ** Mode 
Tab. 2: Details of pregnancy outcome for subjects on the TRF arm compared to the placebo arm

\begin{tabular}{|l|l|c|r|c|}
\hline Pregnancy Outcome & & TRF Arm (n=151) & Placebo Arm (n=148) & $p$ \\
\hline Gestation at Delivery (weeks) ${ }^{*}$ & & $38.31 \pm 0.155$ & $38.44 \pm 0.138$ & 0.538 \\
\hline \multirow{2}{*}{ Onset of Labor, n (\%) } & Spontaneous & $104(68.9)$ & $106(71.6)$ & $37(25.0)$ \\
& Induced & $33(21.9)$ & $103(69.6)$ & \\
\hline \multirow{2}{*}{ Mode of Delivery, n (\%) } & Vaginal & $98(64.9)$ & $31(20.9)$ & \\
\hline Estimated Blood Loss (ml) * & Cesarean section & $39(25.8)$ & $295.82 \pm 9.657$ & 0.337 \\
\hline \multirow{2}{*}{ Gender of Baby, n (\%) } & Male & $315.66 \pm 18.249$ & $84(56.8)$ & \\
\hline Birth Weight (g) $*$ & Female & $66(57.0)$ & $64(43.2)$ & \\
\hline
\end{tabular}

* Mean \pm SEM

on her own accord or prescribed (such as hematinics) were recorded. Compliance to TRF or placebo was monitored by leftover capsules count, and by using a compliance book in which the subject records episodes of missed capsules, if any.

\section{Fetal Monitoring}

First trimester dating sonography was performed, followed by detailed sonography at 18 to 20 weeks to exclude fetal anomaly.

\section{Primary Outcome Measure}

The primary outcome measure was occurrence of PIH. In each case of PIH, the standard investigations that were clinically indicated such as full blood count, renal profile, uric acid and, if indicated, liver function test and coagulation profile, were done to assess severity of disease. Subjects were managed according to standard treatment guidelines for PIH.

\section{Data Analyses}

Data was collected on a standard clinical record format (CRF) and transferred to a Visual Basic database specially designed for this study. Data editing and unblinding of cases in the database was password-protected. Data was analysed using SPSS version 17.0.

\section{Results}

A total of 319 subjects were recruited. Out of this number, $20(6.3 \%)$ subjects dropped out due to various reasons, leaving a final count of 299 subjects who completed the study.

\section{Socio-Demographic Profile}

Of the 299 subjects, 151 were in the TRF arm and 148 in the placebo arm. The socio-demographic profile of these patients is shown in Table 1. The groups were matched for age, body weight, ethnicity and social status, and all subjects were recruited before 16 weeks gestation.

\section{Pregnancy Outcome}

Birth details are tabulated in Table 2. The two groups did not differ significantly in terms of gestation at delivery, onset of labour, mode of delivery, estimated blood loss at delivery, and gender distribution and birth weight of babies.

\section{Incidence of PIH}

The overall incidence of PIH was 5\% (15/299). Four (2.6\%) subjects developed PIH in the TRF arm compared to $11(7.4 \%)$ in the placebo arm. The relative risk of PIH with TRF supplementation was therefore 0.36 (range 0.12-1.09 at $95 \%$ confidence interval), narrowly crossing unity, with a $p$ value of 0.058 .

Only one $(0.7 \%)$ subject developed preeclampsia in the TRF arm compared to five subjects $(3.4 \%)$ in the placebo $\mathrm{arm}$. The relative risk of preeclampsia with TRF supplementation was therefore 0.20 (range $0.02-1.66$ at $95 \%$ confidence interval) but the difference was not statistically significant $(p=0.094)$.

The relative risks of PIH as well as preeclampsia with TRF supplementation versus placebo are shown in Table 3.

\section{Severity of PIH}

The outcome of pregnancy and severity of PIH, which is inclusive of preeclampsia, among subjects on TRF versus placebo was further scrutinized.

Tab. 3: Relative Risk of PIH and preeclampsia with TRF supplementation versus placebo

\begin{tabular}{|l|c|c|c|}
\hline Outcome & $\begin{array}{c}\text { Relative } \\
\text { Risk }\end{array}$ & $\begin{array}{c}95 \% \text { Confidence } \\
\text { Interval }\end{array}$ & $p$ \\
\hline PIH & 0.36 & $0.12-1.09$ & 0.058 \\
\hline Preeclampsia & 0.20 & $0.02-1.66$ & 0.094 \\
\hline
\end{tabular}


Tab. 4: Clinical severity and pregnancy outcome of subjects with PIH in the TRF arm versus the placebo arm

\begin{tabular}{|l|l|c|c|c|}
\hline Parameter & & TRF Arm (n=4) & Placebo Arm $(\mathrm{n}=11)$ & $p$ \\
\hline \multirow{2}{*}{ Highest Blood Pressure (mmHg)* } & Systolic & $155.00 \pm 8.66$ & $149.82 \pm 6.19$ & 0.643 \\
& Diastolic & $100.00 \pm 7.07$ & $99.55 \pm 2.39$ & 0.955 \\
\hline Gestation at Diagnosis (weeks)* & & $37.08 \pm 1.47$ & $38.62 \pm 0.36$ & 0.376 \\
\hline Gestation at Delivery (weeks)* & & $37.78 \pm 1.12$ & $39.08 \pm 0.37$ & 0.334 \\
\hline \multirow{3}{*}{ Onset of Labour, n (\%) } & Spontaneous & $0(0.0)$ & $0(0.0)$ \\
& Induced & $3(75.0)$ & $10(90.9)$ \\
& Elective Caesarean section & $1(25.0)$ & $1(9.1)$ & $7(63.6)$ \\
\multirow{2}{*}{ Mode of Delivery, n (\%) } & Vaginal & $2(50.0)$ & $4(36.4)$ & \\
\hline Estimated Blood Loss (ml)* & Cesarean section & $2(50.0)$ & $350 \pm 40$ & $0.033^{* *}$ \\
\hline Birth Weight (g)* & & $225 \pm 32$ & $3115 \pm 204$ & 0.262 \\
\hline
\end{tabular}

$*$ Mean \pm SEM; ** Statistically significant

Tab. 5: Hematological and biochemical indices of subjects with PIH in the TRF arm versus the placebo arm

\begin{tabular}{|l|c|c|c|}
\hline Hematological / Biochemical Parameter & TRF Arm $(\mathrm{n}=4)$ & Placebo Arm $(\mathrm{n}=11)$ & $p$ \\
\hline Hemoglobin $(\mathrm{mg} / \mathrm{dl})^{*}$ & $12.28 \pm 0.30$ & $11.36 \pm 0.36$ & 0.077 \\
\hline Platelets $\left(\times 10^{9} / \mathrm{L}\right)^{*}$ & $261 \pm 52$ & $298 \pm 24$ & 0.552 \\
\hline Urea $(\mathrm{mmol} / \mathrm{l}) *$ & $4.13 \pm 0.98$ & $3.26 \pm 0.31$ & 0.472 \\
\hline Creatinine $(\mathrm{mmol} / \mathrm{l}) *$ & $56.67 \pm 5.81$ & $64.90 \pm 4.06$ & 0.307 \\
\hline Uric Acid $(\mu \mathrm{mol} / \mathrm{l})^{*}$ & $320 \pm 24$ & $325 \pm 35$ & 0.903 \\
\hline
\end{tabular}

$*$ Mean \pm SEM

Table 4 shows the pregnancy outcome and clinical severity of PIH among affected subjects in both arms of the trial, whereas Table 5 shows the hematological and biochemical indices of these subjects. There was no significant difference in the clinical severity, or the hematological or biochemical indices. The degree of proteinuria could only be quantitatively assessed in terms of 24-hour urine collection in two subjects with preeclampsia. The 24-hour urine protein levels in the subject on TRF and on placebo were $392 \mathrm{mg}$ and $947 \mathrm{mg}$ respectively. Four out of five cases of preeclampsia in the placebo arm had to have pregnancy terminated less than 24 hours after admission, based on the severity of disease, and hence did not have time for 24-hour urine collection to assess protein excretion. Each of these patients had proteinuria of $2+$ to $4+$ on dipstick testing. None of the subjects developed liver impairment or coagulopathy as a result of PIH.

There was no significant difference in pregnancy outcome except for a statistically significantly less blood loss at delivery in the TRF group.

\section{Discussion}

The elusiveness of a definite pathophysiological pathway and treatment modality for PIH other than termination of pregnancy, has resulted in various, generally futile, attempts over the years to look into screening and prophylactic measures for this obstetrically important and potentially fatal disease. This includes diverse drugs such as aspirin, calcium, fish oil, vitamin E and vitamin C (13).

Since submission of the proposal of this study for funding, many other similar studies have been carried out on both sides of the Atlantic, to assess the potential role of antioxidants in the prevention of PIH. However, this study is different from the other studies in two important and significant aspects:

1. This is the first study ever to assess the effect of palm oil vitamin E containing predominantly tocotrienols, rather than soya bean vitamin E containing predominantly tocopherols, a weaker antioxidant, in the prevention of preeclampsia.

2. This study evaluates the effect of palm oil vitamin $E$ alone, rather than in combination with other antioxidants, in the prevention of $\mathrm{PIH}$, unlike the other studies, which combined vitamin $\mathrm{E}$ with vitamin $\mathrm{C}$.

It is important to note that this is the first completed study that uses antioxidant supplementation from early in pregnancy, strictly by 16 weeks gestation. This decision was logical considering the fact that the postulated insult that leads to PIH is failure of the second wave of trophoblastic invasion, which is supposed to occur between 12 and 14 weeks gestation (7). A previous study using aspirin as prophylaxis concluded that any attempt at prophylaxis against PIH should commence before 16 weeks gestation in order to show a positive effect (14).

The manufacturer's recommended dose of TRF was 50-100 mg or 1-2 capsules a day. It was decided to use 
the higher of the two dosages, i.e. $100 \mathrm{mg}$ of TRF or two capsules per day, in view of the large $(50 \%)$ physiological increase in total blood volume in pregnancy (15) that alters the pharmacokinetics of drugs during pregnancy, with resultant dilutional effect. Critiques of the CLASP trial on aspirin (16) postulated that the dose of aspirin used in that trial may have been too low to prevent preeclampsia.

Another factor that influences drug pharmacokinetics is body weight. From the pharmacokinetic point of view, drug dosing is based on body weight rather than Body Mass Index (BMI). To equalize the distribution of body weight between the two arms of the study, subjects were randomized in a stratified manner above and below a body weight of $55 \mathrm{~kg}$, which is the median body weight at gestations of 12-16 weeks in our antenatal population. This was also important in view of the fact that obesity increased the risk for PIH (12).

It may be argued that the ideal population to study in such a clinical trial is one with the highest possible risk of developing PIH. Besides primigravidae, such a population includes pregnant women with previous history of severe $\mathrm{PIH}$, or those with other high risk biomarkers (17). However, in our local setting, multiparous pregnant women tend to book late, well beyond 16 weeks, even in the presence of a previous history of problems. This will make recruitment of a fairly large number of such subjects before 16 weeks gestation, over a reasonably short period of time, quite impossible to achieve, especially in a single hospital. Doppler analysis of the uterine artery was, until the time of this study, an infrequent practice in this hospital, therefore making the skill of this assessment limited to a very small number of personnel.

It has been generally accepted that preeclampsia is a disease of first pregnancy, with a lower incidence in subsequent pregnancies. However, the overall incidence of PIH and preeclampsia in this study was well below the expected incidence for a population of primigravidae. This may be attributable to the relative affluence of the population studied as judged by the average monthly household income of the subjects. The fact that the hospital where this study was conducted was not a free or minimal pay health facility despite being a public entity, made it inevitable that only patients who could afford to pay for their health care sought treatment here. It is a well known fact that PIH is more rampant among the economically deprived.

This unexpectedly low incidence of PIH resulted in difficulty in achieving a statistically significant difference in incidence between the two arms. Nevertheless, there was marked disparity in the incidence between the two arms of this study, in contrast with the VIP trial on tocopherol and Vitamin $\mathrm{C}$ where, out of a total of 100 women at risk of preeclampsia, the incidence of disease in the treatment arm $(17.3 \%)$ was so close to that on the placebo arm $(18.8 \%)$ that it is probably not worthwhile to pursue such studies on a larger scale (18). Having said that, a statistically significant difference in outcome was not achieved in our study, hence the benefit of TRF remains questionable. A larger clinical trial, preferably in a population at higher risk for preeclampsia, is warranted before a firm conclusion can be made regarding TRF.

The smallness of the number of subjects who developed PIH made it impossible to sub-analyze the outcome according to severity of disease. In addition, no one developed severe complications such as HELLP Syndrome or eclampsia. No maternal or perinatal mortality was recorded.

Interestingly, the mean estimated blood loss at delivery was significantly lower in the TRF arm among subjects who developed PIH. However, this is not supported by similar results when the two arms were compared in general (Table 2). Nevertheless, it dispels concerns regarding the anticoagulant properties of Vitamin E, and reassures the continued use of this supplementation without worry of excessive blood loss as a result of its administration.

\section{Conclusion}

This study failed to prove the benefit of antenatal supplementation with TRF from palm oil beyond reasonable doubt. An unexpectedly low incidence of PIH in the sample studied is possibly a contributory factor to the failure. Nevertheless, the novel results from this study suggest that it may be worthwhile to conduct a larger, multicentre trial, in order to assess the benefits of such supplementation in women who are screened to be at high risk based on previous history and socio-demographic attributes, as well as sonographic and biochemical markers. The significance of PIH in terms of maternal and perinatal mortality and morbidity, dictate that such a study should be considered a priority.

\section{Acknowledgements}

Funding through IRPA Grant (06-02-02-0136) from the Ministry of Science Technology and Environment under RMK-7, and contribution of TRF supplements and placebo capsules from Golden Hope Bioganics Sdn Bhd, are gratefully acknowledged. The researchers would also like to thank Anson Ismail, Rosnani Ismail, Naizaithull Fahya Mujammil, Azri Harun, Yong Nor Azizah, Niefaizal Abdul Hammid, and Nor Azliza Wani Abdul Aziz, for their invaluable assistance throughout the study. Our deepest appreciation also goes to all women who participated, without whom this study would have been impossible.

\section{References}

\footnotetext{
1. Villar K, Say L, Gulmezoglu AM, Merialdi M, Lindheimer MD, Betran AP, Piaggio G. Eclampsia and pre-eclampsia: a health problem for 2000 years. In: Critchley H, MacLean AB, Poston L, Walker JJ, eds. Pre-eclampsia. London: RCOG Press, 2003: 189-207.

2. Tan KH, Kwek K, Yeo GSH. Epidemiology of pre-eclampsia and eclampsia at the KK Women's and Children's Hospital, Singapore. Singapore Med J 2006; 47(1): 48 .

3. Pitakkarnkul S, Phaloprakarn C, Wiriyasirivaj B, Manusirivithaya S, Tangjitgamol S. Seasonal variation in the prevalence of preeclampsia. J Med Assoc Thai 2011; 94(11): 1293-8.

4. Brown MA, Lindheimer MD, de Swiet M, van Assche A, Moutquin JM. The classi-
} 
fication and diagnsosis of the hypertensive disorders of pregnancy: statement from the International Society for the Study of Hypertension in Pregnancy (ISSHP). Hypertension in Pregnancy 2001; 20: IX-XIV.

5. Hubel CA. Oxidative stress in the pathogenesis of preeclampsia. Proc Soc Exp Biol Med 1999; 222: 222-235

6. Raijmakers MTM, Dechend R, Poston L. Oxidative stress and preeclampsia: rationale for antioxidant clinical trials. Hypertension 2004; 44: 374-380.

7. Roberts JM, Taylor RN, Musci TJ et al. Preeclampsia: and endothelial cell disorder. Am J Obstet Gynecol 1989; 161: 1200-1204.

8. Hubel CA, Roberts JM, Taylor RN, et al. Lipid peroxidation in pregnancy: new perspectives on preeclampsia. Am J Obstet Gynecol 1989; 161: 1025-1034

9. Morris JM, Gopaul NK, Endresen MJ et al. Circulating markers of oxidative stress are raised in normal pregnancy and pre-eclampsia. Br J Obstet Gynaecol 1998; 105(11): 1195-1199.

10. Gulmezoglu AM, Hofmeyr GJ, Oosthuisen MM. Antioxidants in the treatment of severe pre-eclampsia: an explanatory randomized controlled trial. Br J Obstet Gynaecol 1997; 104(6): 689-696.

11. Serbinova E, Kagan V, Han D, Packer L. Free radical recycling and intramembran mobility in the antioxidant properties of alpha-tocopherol and alpha-tocotrienol. Free Rad Biol Med 1991; 10: 263-275.
12. The Royal College of Obstetricians and Gynaecologists \& Royal College of Midwives. Hypertension in pregnancy: the management of hypertensive disorders during pregnancy. NICE Clinical Guideline 2011.

13. Duley L. Pre-eclampsia and the hypertensive disorders of pregnancy. Br Med Bulletin 2003; 67(1): 161 .

14. Hermida RC, Ayala DE, Fernandez JR, Mojon A, Alonso I, Silva I, Ucieda R, Codesido J, Iglesias M. Administration time-dependent effects of aspirin in women at differing risk for preeclampsia. Hypertension 1999; 34: 1016-1023.

15. Pirani BBK, Campbell DM \& MacGillivray I. Plasma volume in normal first pregnancy. J Obstet Gynaecol Br Commonwealth 1973; 80: 884-887.

16. CLASP (Collaborative Low-dose Aspirin Study in Pregnancy) Collaborative Group. CLASP: a randomised trial of low-dose aspirin for the prevention and treatment of pre-eclampsia among 9364 pregnant women. Lancet 1994; 343: 619-629.

17. Poon LCY, Akolekar R, Lachmann R, Beta J, Nicolaides KH. Hypertensive disorders in pregnancy: screening by biophysical and biochemical markers at 11-13 weeks. Ultrasound Obstet Gynecol 2010; 35: 662-670.

18. Poston L, Briley AL, Seed PT, Kelly FJ, Shennan AH, Vitamins in Pre-eclampsia (VIP) Trial Consortium. Vitamin C and vitamin $\mathrm{E}$ in pregnant women at risk for pre-eclampsia (VIP Trial): randomised placebo-controlled trial. Lancet 2006; 367(9517): 1145-54.

Received: 04/11/2012

Accepted in revised form: 24/09/2013

\section{Corresponding Author:}

Professor Dr Zaleha Abdullah Mahdy, Department of Obstetrics and Gynaecology, Faculty of Medicine, Universiti Kebangsaan Malaysia, Jalan Yaacob Latif, 56000 Cheras, Kuala Lumpur, Malaysia; e-mail: zaleha@ppukm.ukm.edu.my 